\title{
What are the barriers to improving care for patients with sepsis?
}

\author{
Authors: Sarah Cowan, Jonathan Holland, Andrew Kane and Ian Frost
}

\section{Aims}

The aims of this study were to identify what junior doctors perceive to be the barriers to improving care for patients with sepsis and to assess the ability of junior doctors to recognise sepsis.

\section{Methods}

A questionnaire was distributed to all foundation year 1 and 2 doctors attending a weekly teaching session, with 46 respondents. Participants were asked to rank six possible barriers to delivering sepsis care in order of importance. The questionnaire also tested knowledge of sepsis and the 'Sepsis Six' (a set of basic interventions that have been shown to reduce mortality from sepsis by $40 \%$ ).

\section{Results}

Junior doctors perceived the greatest barriers in treating sepsis to be (in order from most to least important): 1. time pressure on call; 2 . knowledge and recognition by non-medical staff; 3 . time to delivery of antibiotics and fluids once prescribed; 4 . availability of senior support; 5 . concerns about correct choice of antibiotics; 6 . their own knowledge and recognition of sepsis.

Only $4 \%$ were able to list the Systemic Inflammatory Response (SIRS) criteria and only 50\% could define sepsis. A common misconception was that hypotension, oliguria and hypoxia were SIRS criteria, whereas in fact they indicate severe sepsis with organ dysfunction. $46 \%$ could list the 'Sepsis Six'.

Following further teaching on sepsis, $38 \%$ could list the SIRS criteria, $87 \%$ correctly defined sepsis, and $92 \%$ could list the 'Sepsis Six'.

\section{Conclusions}

The greatest perceived barrier to improving sepsis care was time pressure on call. This reflects the pressure that junior doctors face both on the wards and in the emergency department, particularly out of hours. The time taken to deliver antibiotics and fluids once prescribed may reflect the pressure that nurses are working under in busy clinical environments.

Knowledge of sepsis amongst junior doctors was poor. Additionally there was a lack of insight, with junior doctors not realising that limitations in their own knowledge were an important barrier to improving care.

If patients with sepsis are to receive timely care, then sufficient personnel and resources need to be in place to ensure that antibiotics and intravenous fluids can be delivered promptly. Our results show that continuing education of medical staff is also necessary if earlier recognition and treatment of sepsis are to be achieved. We have subsequently delivered further teaching on sepsis to both nursing and medical staff in our hospital, with a significant improvement in knowledge of sepsis.

\section{Conflict of interest statement}

No conflict of interest declared by any author. 